\begin{tabular}{ll} 
Prof. Dr. med. \\
H. S. Fü̈ßI \\
$\begin{array}{ll}\text { Isar-Amper- } \\
\text { Klinikum, } \\
\text { Kl. München-Ost, } \\
\text { Haar }\end{array}$ \\
$\begin{array}{ll}\text { K. Holzgreve } \\
\text { Internist, } \\
\text { Kardiologische } \\
\text { Praxis, München }\end{array}$ \\
\hline
\end{tabular}

\title{
Herzinsuffiziente Diabetiker: bessere Prognose bei höherem $\mathrm{HbA}_{1 c}$
}

\section{Die Prognose von Diabetikern mit fortgeschrittener Herzinsuffizienz ist umso schlechter, je niedriger der $\mathrm{HbA}_{1 c}$-Wert ist.}

- In einer retrospektiven Analyse wurden die Daten von 845 Patienten ausgewertet (mittleres Alter 55 Jahre), die zwischen 1999 und 2010 zur kardiologischen Untersuchung und evtl. Herztransplantation überwiesen worden waren, in der Regel also an einer schweren Herzinsuffizienz (78\% NYHA III und IV) litten. 358 der Patienten waren Diabetiker und 487 Nicht-Diabetiker. Sie wurden umfangreich untersucht und in Quartilen der $\mathrm{HbA}_{1 c}$ eingeteilt. Als primärer Endpunkt wurden Tod und Notwendigkeit zur Herztransplantation nach zwei Jahren definiert.

Die retrospektive Auswertung ergab für Diabetiker: Je höher das $\mathrm{HbA}_{1 c}$ in den Quartilen, umso häufiger blieben die Patienten von Tod und Transplantation verschont (s. Tab.). Oder umgekehrt: je „besser" die Stoffwechseleinstellung bzw. je niedriger das $\mathrm{HbA}_{1 \mathrm{c}}$, umso häufiger waren Tod oder Herztransplantation. Nach Berücksichtigung zahlreicher Einflussfaktoren errechnete sich für jeden absoluten Anstieg des

\begin{tabular}{|l|l|c|}
\hline Tabelle & & \\
\hline Quartile & $\mathrm{HbA}_{1 c}$ & 2-Jahres-Überlebensrate ohne Herztransplantation \\
\hline 1 & $\leq 5,6$ & $47,9 \%$ \\
\hline 2 & $5,7-6,0$ & $41,5 \%$ \\
\hline 3 & $6,1-6,5$ & $60,7 \%$ \\
\hline 4 & $\geq 6,6$ & $65,3 \%$ \\
\hline
\end{tabular}

Anteil (\%) von herzinsuffizienten Diabetikern ohne Endpunkt innerhalb von zwei Jahren in Abhängigkeit von Quartilen des $\mathrm{HbA}_{1 \mathrm{c}}$-Wertes

$\mathrm{HbA}_{1 \mathrm{c}}$ um 1\% (also z.B. von 6 auf 7\%) eine Abnahme für Tod und Herztransplantation um 15\%. Bei den Nicht-Diabetikern ergab sich keine Korrelation zwischen dem genannten Endpunkt und dem $\mathrm{HbA}_{1 c}$, bei naturgemäß niedrigeren Werten und einem engeren Bereich.

\section{Kommentar}

Einerseits gilt als gesichert, dass Diabetiker umso häufiger eine Herzinsuffizienz entwickeln, je höher das $\mathrm{HbA}_{1 c}$ ist. Andererseits gibt es kontroverse Befunde zum Verlauf einer bereits bestehenden Herzinsuffizienz in Abhängigkeit von der Höhe des $\mathrm{Hb}_{1} \mathrm{~A}_{1 \mathrm{c}}$. Das Ergebnis der vorliegenden Studie kommt nicht überraschend. Mehrere Studien wie z.B. ACCORD haben gezeigt, dass durch strenge Blutzuckereinstellung bei Diabetikern ma- krovaskuläre Komplikationen und Mortalität nicht verhindert, möglicherweise sogar ungünstig beeinflusst werden. Zum gegenwärtigen Zeitpunkt empfehlen die Leitlinien der Fachgesellschaften, die antidiabetische Therapie und die Zielwerte für Blutzucker und $\mathrm{HbA}_{1 c} \mathrm{Zu}$ "individualisieren", d.h. bei Diabetikern mit hohem kardiovaskulärem Risiko und - wie im vorliegenden Fall gezeigt - mit Herzinsuffizienz $\mathrm{HbA}_{1 c}$-Werte zwischen etwa 7 und $8 \%$ anzustreben.

H. HolzGREVE -

- G. S. Tomova et al.

Relation between hemoglobin A1c and outcomes in heart failure patients with and without diabetes mellitus. Amer. J. Cardiol. online 27 March 2012 